\title{
ON SEVERAL TYPES OF CONTINUOUS FUNCTIONS INDUCED BY GENERALIZED $w$-OPEN SETS IN ASSOCIATED $w$-SPACES
}

\author{
Won Keun Min ${ }^{1 \S}$, Young Key Kim² \\ ${ }^{1}$ Department of Mathematics \\ Kangwon National University \\ Chuncheon, 200-701, KOREA \\ ${ }^{2}$ Department of Mathematics \\ Myong.Ji University \\ Yongin, 449-728, KOREA
}

\begin{abstract}
The purpose of this paper is to introduce the notions of $g w_{\tau^{-} \text {-continuous, } g w_{\tau^{-}}^{*}}$ continuous, $g w_{\tau}$-irresolute, and $g w_{\tau}^{*}$-irresolute functions by using $g w_{\tau}$-open sets between an associated $w_{\tau}$-spaces, and to study its characterizations and the relationships among them.
\end{abstract}

AMS Subject Classification: 54A05, 54B10, 54C10

Key Words: associated $w_{\tau^{-}}$-space, generalized $w_{\tau^{-}}$open $\left(g w_{\tau^{-}}\right.$open $), g w_{\tau^{-}}$continuous, $g w_{\tau^{-}}^{*}$ continuous, $g w_{\tau}$-irresolute, $g w_{\tau}^{*}$-irresolute

\section{Introduction}

Siwiec [13] introduced the notions of weak neighborhoods and weak base in a topological space. We introduced the weak neighborhood systems defined by using the notion of weak neighborhoods in [8]. The weak neighborhood system induces a weak neighborhood space which is independent of neighborhood spaces [2] and general topological spaces [1].

Received: $\quad$ September 2, 2016

Revised: September 14, 2016

Published: November 4, 2016

$\S_{\text {Correspondence author }}$ (c) 2016 Academic Publications, Ltd. url: www.acadpubl.eu 
The notions of weak structure, $w$-space, $W$-continuity and $W^{*}$-continuity were investigated in [9]. In fact, the set of all $g$-closed subsets [3] in a topological space is a kind of weak structure. Moreover, In [10], we introduced the notion of an associated weak space (simply, associated $w_{\tau}$-space) containing a given topology $\tau$. In the similar way introduced by Levine [3] in topological spaces, we introduced the notion of generalize $w_{\tau}$-open sets (generalize $w_{\tau}$-closed sets) [11] in an associated weak space $w_{\tau}$ and studied its properties. In this paper, we are going to introduce the notions of $g w_{\tau}$-continuous, $g w_{\tau}^{*}$-continuous, $g w_{\tau}$-irresolute and $g w_{\tau}^{*}$-irresolute functions between an associated $w_{\tau}$-spaces by using generalize $w_{\tau}$-open sets $\left(g w_{\tau}\right.$-open sets), and to study its characterizations and the relationships among them.

\section{Preliminaries}

Definition $2.1([9])$. Let $X$ be a nonempty set. A subfamily $w_{X}$ of the power set $P(X)$ is called a weak structure on $X$ if it satisfies the following:

(1) $\emptyset \in w_{X}$ and $X \in w_{X}$.

(2) For $U_{1}, U_{2} \in w_{X}, U_{1} \cap U_{2} \in w_{X}$.

Then the pair $\left(X, w_{X}\right)$ is called a $w$-space on $X$. Then $V \in w_{X}$ is called a $w$-open set and the complement of a $w$-open set is a $w$-closed set.

The collection of all $w$-open sets (resp., $w$-closed sets) in a $w$-space $X$ will be denoted by $W O(X)$ (resp., $W C(X)$ ). We set $W(x)=\{U \in W O(X): x \in U\}$.

Let $S$ be a subset of a topological space $X$. The closure (resp., interior) of $S$ will be denoted by $c l S$ (resp., int $S$ ). A subset $S$ of $X$ is called a preopen set [6] (resp., $\alpha$-open set [12], semi-open [4]) if $S \subset \operatorname{int}(\operatorname{cl}(S))$ (resp., $S \subset$ $\operatorname{int}(\operatorname{cl}(\operatorname{int}(S))), S \subset \operatorname{cl}(\operatorname{int}(S)))$. The complement of a preopen set (resp., $\alpha-$ open set, semi-open) is called a preclosed set (resp., $\alpha$-closed set, semi-closed). The family of all preopen sets (resp., $\alpha$-open sets, semi-open sets) in $X$ will be denoted by $P O(X)$ (resp., $\alpha(X), S O(X)$ ). We know the family $\alpha(X)$ is a topology finer than the given topology on $X$.

Moreover, a subset $S$ of $X$ is said to be $g$-closed [3] if $\operatorname{cl}(A) \subset U$ whenever $A \subset U$ and $U$ is open in $X$.

Then the family $G O(X)=\{U \subseteq X$ : $U$ is $g$-open $\}, O(X)=\{U \subseteq X$ : $U$ is open $\}$ and $C L(X)=\{F \subseteq X: F$ is closed $\}$ are all weak structures on $X$. But $P O(X), G P O(X)$ and $S O(X)$ are not weak structures on $X$. A subfamily $m_{X}$ of the power set $P(X)$ of a nonempty set $X$ is called a minimal structure on $X[5]$ if $\emptyset \in w_{X}$ and $X \in w_{X}$. Thus clearly every weak structure is a minimal structure. 
Let $X$ be a nonempty set and let $(X, \tau)$ be a topological space. A subfamily $w$ of the power set $P(X)$ is called an associated weak structure (simply, $w_{\tau}$ ) [10] on $X$ if $\tau \subseteq w$ and $w$ is a weak structure. Then the pair $\left(X, w_{\tau}\right)$ is called an associated $w$-space with $\tau$.

Let $\left(X, w_{\tau}\right)$ be an associated $w$-space with a topology $\tau$ and $A \subseteq X$. Then $A$ is called a generalized $w_{\tau}$-closed set (simply, $g w_{\tau}$-closed set) [11] if $c l(A) \subseteq U$, whenever $A \subseteq U$ and $U$ is $w$-open. Then the union of two $g w_{\tau}$-closed sets is a $g w_{\tau}$-closed set, but the intersection of two $g w_{\tau}$-closed sets is not always $g w_{\tau}$-closed. And $A$ is called a generalized $w_{\tau}$-open set (simply, g $w_{\tau}$-open set) if $X-A$ is $g w_{\tau}$-closed.

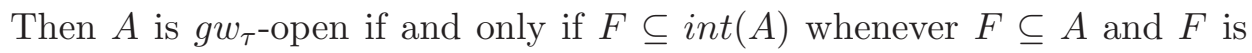
$w$-closed.

For a subset $A$ of $X, g w_{\tau}$-closure of $A$ and $g w_{\tau}$-interior of $A$ are defined as the following:

(1) $g w_{\tau} C(A)=\cap\left\{F: A \subseteq F, F\right.$ is $g w_{\tau^{-}}$closed $\}$.

(2) $g w_{\tau} I(A)=\cup\left\{U: U \subseteq A, U\right.$ is $\left.g w_{\tau^{-o p e n}}\right\}$.

Theorem $2.2([11])$. Let $\left(X, w_{\tau}\right)$ be an associated $w$-space with a topology $\tau$ and $A \subseteq X$.

(1) If $A$ is $g w_{\tau}$-open $\left(g w_{\tau}\right.$-closed), then $g w_{\tau} I(A)=A\left(g w_{\tau} C(A)=A\right)$.

(2) If $A \subseteq B$, then $g w_{\tau} I(A) \subseteq g w_{\tau} I(B) ; g w_{\tau} C(A) \subseteq g w_{\tau} C(B)$.

(3) $g w_{\tau} C(X-A)=X-g w_{\tau} I(A) ; g w_{\tau} I(X-A)=X-g w_{\tau} C(A)$.

(4) $x \in g w_{\tau} I(A)$ if and only if there exists a $g w_{\tau}$-open set $U$ containing $x$ such that $U \subseteq A$.

(5) $x \in g w_{\tau} C(A)$ if and only if $A \cap V \neq \emptyset$ for all $g w_{\tau}$-open set $V$ containing $x$.

(6) $\emptyset=g w_{\tau} C(\emptyset) ; \quad X=g w_{\tau} I(X)$.

(7) $g w_{\tau} I(A) \subseteq A \subseteq g w_{\tau} C(A)$.

(8) $g w_{\tau} C(A \cup B)=g w_{\tau} C(A) \cup g w_{\tau} C(B) ; \quad g w_{\tau} I(A \cap B)=g w_{\tau} I(A) \cap$ $g w_{\tau} I(B)$.

(9) $g w_{\tau} C\left(g w_{\tau} C(A)\right)=g w_{\tau} C(A) ; g w_{\tau} I\left(g w_{\tau} I(A)\right)=g w_{\tau} I(A)$.

\section{3. $g w_{\tau}$-Continuity; $g w_{\tau}^{*}$-Continuity}

Definition 3.1. Let $f:\left(X, w_{\tau}\right) \rightarrow\left(Y, w_{\mu}\right)$ be a function in two associated $w$-spaces with $\tau$ and $\mu$. Then $f$ is said to be

(1) $g w_{\tau}$-continuous if for $x \in X$ and for each open set $V$ containing $f(x)$, there is a $g w_{\tau}$-open set $U$ containing $x$ such that $f(U) \subseteq V$ : 
(2) $g w_{\tau}^{*}$-continuous if for every open set $V$ in $Y, f^{-1}(V)$ is a $g w_{\tau}$-open set in $X$.

Obviously we obtain the following theorem:

Theorem 3.2. Every $g w_{\tau}^{*}$-continuous function is $g w_{\tau}$-continuous.

The following example supports that the converse of the above theorem is not true in general.

Example 3.3. Let $X=\{a, b, c, d\}$, a topology $\tau=\{\emptyset,\{b\}, X\}$ and a $w$-structure $w_{X}=\{\emptyset,\{a, c\},\{a\},\{b\},\{c\},\{a, d\}, X\}$ in $X$. Note: The set of all $g w_{\tau}$-closed sets is $\{\emptyset, X,\{a, b\},\{b, c\},\{b, d\},\{a, b, c\},\{a, b, d\},\{a, c, d\},\{b, c, d\}\}$.

Now, consider a function $f:\left(X, w_{\tau}\right) \rightarrow\left(X, w_{\tau}\right)$ defined by $f(a)=f(c)=a$; $f(b)=f(d)=b$. For $b, d \in X$ and for the only open set $V=\{b\}$ containing $f(b)=f(d)$, since $A=\{b\}$ and $B=\{d\}$ are $g w_{\tau}$-open sets containing $b$ and $d$, respectively, so obviously $f$ is $g w_{\tau}$-continuous. But $f^{-1}(V)=\{b, d\}=A \cup B$ is

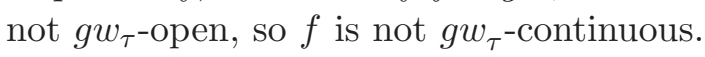

We recall that: Let $f:\left(X, w_{\tau}\right) \rightarrow(Y, \mu)$ be a function on an associated $w$-space with $\tau$ and a topological space $(Y, \mu)$. Then $f$ is said to be

(1) WO-continuous [10] if for $x \in X$ and for each open set $V$ containing $f(x)$, there is a $w$-open set $U$ containing $x$ such that $f(U) \subseteq V$;

(2) WK-continuous [10] if for every open set $V$ in $Y, f^{-1}(V)$ is a w-open set in $X$.

Obviously, the following things are obtained:

Theorem 3.4. (1) Every WO-continuous function is $g w_{\tau}$-continuous.

(2) Every $W K$-continuous function is $g w_{\tau}^{*}$-continuous.

The following example supports that the converses of the above theorem are not true in general.

Example 3.5. (1) Consider the function $f$ defined in Example 3.3. Then the $g w_{\tau}$-continuous function $f$ is not $W O$-continuous since for $d \in X$ and for the only open set $V=\{b\}$ containing $f(d)$, there is no any $w$-open set $U$ containing $d$ such that $f(U) \subseteq V$.

(2) Let $X=\{a, b, c, d\}$, a topology $\tau=\{\emptyset,\{b\}, X\}$ and a $w$-structure $w_{X}=$ $\{\emptyset,\{a\},\{b\},\{c\},\{a, d\}, X\}$ in $X$. Consider a function $f:\left(X, w_{\tau}\right) \rightarrow\left(X, w_{\tau}\right)$ defined by $f(a)=f(c)=a ; f(b)=f(d)=b$. Then $f^{-1}(\{b\})=\{b, d\}$ is $g w_{\tau}$-open, so $f$ is $g w_{\tau}^{*}$-continuous. But since $\{b, d\}$ is not $w$-open, $f$ is not $W K$-continuous.

Remark 3.6. For a function from an associated $w_{\tau}$-space with $\tau$ to a topological space, we have the following diagram: 


$$
\begin{aligned}
& \text { Continuity } \Rightarrow W K \text {-continuity } \Rightarrow W O \text {-continuity } \\
& \Downarrow \\
& g w_{\tau}^{*} \text {-continuity } \Rightarrow g w_{\tau^{-}} \text {continuity }
\end{aligned}
$$

Theorem 3.7. Let $f:\left(X, w_{\tau}\right) \rightarrow(Y, \mu)$ be a function on an associated $w$-space with $\tau$ and a topological space $(Y, \mu)$. Then the following statements are equivalent:

(1) $f$ is $g w_{\tau}$-continuous.

(2) $f\left(g w_{\tau} C(A)\right) \subseteq c l(f(A))$ for $A \subseteq X$.

(3) $g w_{\tau} C\left(f^{-1}(V)\right) \subseteq f^{-1}(\operatorname{cl}(V))$ for $V \subseteq Y$.

(4) $f^{-1}(\operatorname{int}(V)) \subseteq g w_{\tau} I\left(f^{-1}(V)\right)$ for $V \subseteq Y$

Proof. $(1) \Rightarrow(2)$ Let $x \in g w_{\tau} C(A)$. Suppose that $f(x)$ is not in $c l(f(A))$; then there exists an open set $V$ containing $f(x)$ such that $V \cap f(A)=\emptyset$. By $g w_{\tau}$-continuity, there is a $g w_{\tau}$-open set $U$ containing $x$ such that $f(U) \subseteq V$, and so $f(U) \cap f(A)=\emptyset$. Hence $U \cap A=\emptyset$, which is a contradiction to $x \in g w_{\tau} C(A)$. This implies that $f\left(g w_{\tau} C(A)\right) \subseteq c l(f(A))$.

$(2) \Rightarrow(3)$ Let $A=f^{-1}(B)$ for $B \subseteq Y$. From (2), it follows $f\left(g w_{\tau} C(A)\right) \subseteq$ $c l(f(A))=c l\left(f\left(f^{-1}(B)\right)\right) \subseteq c l(B)$. It implies that $g w_{\tau} C\left(f^{-1}(B)\right) \subseteq f^{-1}(c l(B))$.

$(3) \Rightarrow(4)$ By Theorem 2.2, it is obvious.

$(4) \Rightarrow(1)$ Let $V$ be any open set containing $f(x)$ for each $x \in X$. Then since $f(x) \in \operatorname{int}(V)$, and by (4), $x \in f^{-1}(\operatorname{int}(V)) \subseteq g w_{\tau} I\left(f^{-1}(V)\right)$. There exists a $g w_{\tau}$-open set $U$ such that $x \in U \subseteq g w_{\tau} I\left(f^{-1}(V)\right) \subseteq f^{-1}(V)$. Hence, $f$ is $g w_{\tau}$-continuous.

Corollary 3.8. Let $f:\left(X, w_{\tau}\right) \rightarrow(Y, \mu)$ be a function on an associated $w$-space with $\tau$ and a topological space $(Y, \mu)$. Then the following statements are equivalent:

(1) $f$ is $g w_{\tau}$-continuous.

(2) $f^{-1}(V)=g w_{\tau} I\left(f^{-1}(V)\right)$ for every open set $V \in Y$.

(3) $f^{-1}(B)=g w_{\tau} C\left(f^{-1}(B)\right)$ for every closed set $B \subseteq Y$.

Proof. From Theorem 2.2, it is obvious.

Theorem 3.9. Let $f:\left(X, w_{\tau}\right) \rightarrow(Y, \mu)$ be a function on an associated $w$-space and a topological space $(Y, \mu)$. Then $f$ is $g w_{\tau}^{*}$-continuous if and only if for every closed set $F$ in $Y, f^{-1}(F)$ is $g w_{\tau}$-closed in $X$.

Proof. It is obvious. 
Let $\left(X, w_{\tau}\right)$ be an associated $w$-space with a topology $\tau$. Let $g W_{\tau}(x)$ denote the set of all $g w_{\tau}$-open set containing $x$ in $X$, and $O(x)$ denote the set of all open set containing $x$ in $X$.

A collection $\mathcal{H}$ of subsets of $X$ is called an m-family [7] on $X$ if $\cap \mathcal{H} \neq \emptyset$. Let $\mathcal{H}$ be an $m$-family on $X$. Then we say that an $m$-family $\mathcal{H} g w_{\tau}$-converges to $x \in X$ if $\mathcal{H}$ is finer than $g W_{\tau}(x)$ i.e., $g W_{\tau}(x) \subseteq \mathcal{H}$. Let $f: X \rightarrow Y$ be a function; then it is obvious $f(\mathcal{H})=\{f(F): F \in \mathcal{H}\}$ is an $m$-family on $Y$. If $\mathcal{F}$ is a filter base, we denote by $\langle\mathcal{F}\rangle$ the filter generated by $\mathcal{H}$.

Theorem 3.10. Let $f:\left(X, w_{\tau}\right) \rightarrow(Y, \mu)$ be a function on an associated $w_{\tau}$-space and a topological space $(Y, \mu)$. Then if $f$ is $g w_{\tau}$-continuous, then for an $m$-family $\mathcal{H} g w_{\tau}$-converging to $x \in X$, a filter $\langle f(\mathcal{H})>$ converges to $f(x)$.

Proof. Suppose $f$ is $g w_{\tau}$-continuous and $\mathcal{H}$ is an $m$-family $g w_{\tau}$-converging to $x \in X$. By $g w_{\tau}$-continuity, for an open set $V$ containing $f(x)$, there exists a $g w_{\tau}$-open set $U$ containing $x$ such that $f(U) \subseteq V$. Since $f\left(g W_{\tau}(x)\right) \subseteq f(\mathcal{H})$, $V \in<f(\mathcal{H})>$ i.e., $O(f(x)) \subseteq<f(\mathcal{H})>$. Hence the filter $<f(\mathcal{H})>$ converges to $f(x)$.

Theorem 3.11. Let $f:\left(X, w_{\tau}\right) \rightarrow(Y, \mu)$ be a bijective function in an associated $w$-space and a topological space $(Y, \mu)$. Then $f$ is $g w_{\tau}^{*}$-continuous iff for an $m$-family $\mathcal{H} g w_{\tau}$-converging to $x \in X$, the filter $\langle f(\mathcal{H})\rangle$ converges to $f(x)$.

Proof. Suppose $f$ is $g w_{\tau}^{*}$-continuous and $\mathcal{H}$ is an $m$-family $g w_{\tau}$-converging to $x \in X$. By hypothesis and surjectivity, we get $O(f(x)) \subseteq f\left(g W_{\tau}(x)\right) \subseteq f(\mathcal{H})$, so that a filter $\langle f(\mathcal{H})>$ converges to $f(x)$.

For the converse, let $U \in O(f(x))$ for $U \subset Y$. Since the family $g W_{\tau}(x)$ clearly $g w_{\tau}$-converges to $x$, by hypothesis, we get $O(f(x)) \subseteq<f\left(g W_{\tau}(x)\right)>$ for $x \in X$. From $f$ is injectivity, it follows $f^{-1}(U) \in g W_{\tau}(x)$.

Corollary 3.12. Let $f:\left(X, w_{\tau}\right) \rightarrow(Y, \mu)$ be a function in an associated $w$-space and a topological space $(Y, \mu)$. If $g w_{\tau}=w$, then $f$ is $W K$-continuous iff for an $m$-family $\mathcal{H} w$-converging to $x \in X$, the filter $\langle f(\mathcal{H})\rangle$ converges to $f(x)$.

\section{4. $g w_{\tau}$-Irresolute; $g w_{\tau}^{*}$-Irresolute}

Definition 4.1. Let $f:\left(X, w_{\tau}\right) \rightarrow\left(Y, w_{\mu}\right)$ be a function on two associated $w$-spaces with $\tau$ and $\mu$. Then $f$ is said to be $g w_{\tau}^{*}$-irresolute if for every $g w_{\tau^{-}}$-open 
set $V$ in $Y, f^{-1}(V)$ is $g w_{\tau^{-}}$open in $X$.

Theorem 4.2. Let $f:\left(X, w_{\tau}\right) \rightarrow\left(Y, w_{\mu}\right)$ be a function on two associated $w$-spaces with $\tau$ and $\mu$. $f$ is $g w_{\tau}^{*}$-irresolute if and only if for every $g w_{\mu}$-closed set $F$ in $Y, f^{-1}(F)$ is $g w_{\tau}$-closed in $X$.

Proof. It is obvious.

Theorem 4.3. Every $g w_{\tau}^{*}$-irresolute function is $g w_{\tau}^{*}$-continuous.

From he following example, we can can that the converse of the above theorem is not true in general.

Example 4.4. Let $X=\{a, b, c, d\}$ and a topology $\tau=\{\emptyset,\{a, b\}, X\}$. Consider a $w$-structures $w=\{\emptyset,\{a, b\},\{b\},\{b, c\}, X\}$ in $X$. Let $f:\left(X, w_{\tau}\right) \rightarrow$ $\left(X, w_{\tau}\right)$ be a function defined as follows $f(a)=b ; f(b)=a ; f(c)=d: f(d)=c$. Then $f$ is $g w_{\tau}^{*}$-continuous by Theorem 3.9, since $f^{-1}(\{c, d\})=\{c, d\}$ for the only closed set $\{c, d\}$ in $(X, \tau)$. But for a $g w_{\tau}$-closed set $\{a, d\}$ in an associated $w$-space, $f^{-1}(\{a, d\})=\{b, c\}$ is not $g w_{\tau}$-closed in the associated $w$-space. So, by Theorem 4.3, $f$ is not $g w_{\tau}^{*}$-irresolute.

Theorem 4.5. Let $f:\left(X, w_{\tau}\right) \rightarrow\left(Y, w_{\mu}\right)$ be a bijective function on two associated $w$-spaces with $\tau$ and $\mu$. Then $f$ is $g w_{\tau}^{*}$-irresolute iff for an $m$-family $\mathcal{H} g w_{\tau}$-converging to $x \in X, f(\mathcal{H}) g w_{\mu}$-converges to $f(x)$.

Proof. Suppose $f$ is $g w_{\tau}^{*}$-irresolute and $\mathcal{H}$ is an $m$-family $g w_{\tau}$-converging to $x \in X$. Then $f\left(g W_{\tau}(x)\right) \subseteq f(\mathcal{H})$ and from surjectivity of $f, g W_{\mu}(f(x)) \subseteq$ $f\left(g W_{\tau}(x)\right) \subseteq f(\mathcal{H})$. So, $f(\mathcal{H}) g w_{\mu}$-converges to $f(x)$.

Conversely, let $U \in g W_{\mu}(f(x))$ for $U \subseteq Y$. Since $g W_{\tau}(x) g w_{\tau}$-converges to $x$, by hypothesis, we get $g W_{\mu}(f(x)) \subseteq f\left(g W_{\tau}(x)\right)$ for $x \in X$. From injectivity of $f$, we have $f^{-1}(U) \in g W_{\tau}(x)$.

Definition 4.6. Let $f:\left(X, w_{\tau}\right) \rightarrow\left(Y, w_{\mu}\right)$ be a function on two associated $w$-spaces with $\tau$ and $\mu$. Then $f$ is said to be $g w_{\tau}$-irresolute if for $x \in X$ and for each $g w_{\mu}$-open set $V$ containing $f(x)$, there is $g w_{\tau}$-open set $U$ containing $x$ such that $f(U) \subseteq V$.

Theorem 4.7. (1) Every $g w_{\tau}^{*}$-irresolute function is $g w_{\tau}$-irresolute.

(2) Every $g w_{\tau}$-irresolute is $g w_{\tau}$-continuous.

The following example supports that the converses of the above theorem are not true in general.

Example 4.8. (1) In Example 3.3, we can easily explain that the function $f$ is $g w_{\tau}$-irresolute but not $g w_{\tau}^{*}$-irresolute. 
(2) Let $X=\{a, b, c, d\}$ and a topology $\tau=\{\emptyset,\{a, b\}, X\}$. Consider a $w$ structures $w=\{\emptyset,\{a, b\},\{b\},\{b, c\},\{a, b, d\}, X\}$ in $X$. Note that the following all sets are $g w_{\tau}$-open: $\emptyset,\{a\},\{b\},\{d\},\{a, b\},\{b, d\}, X$.

Let $f:\left(X, w_{\tau}\right) \rightarrow\left(X, w_{\tau}\right)$ be a function defined as follows $f(a)=b ; f(b)=$ $a ; f(c)=f(d)=d$. Then obviously $f$ is $g w_{\tau}$-continuous. For $c \in X$ and for a $g w_{\tau}$-open set $V=\{d\}$ containing $f(c)=d$, there is no a $g w_{\tau}$-open set $U$ containing $c$ such that $f(U) \subseteq V=\{d\}$. So, $f$ is not $g w_{\tau}$-irresolute.

From Remark 3.6 and the above theorems, we have the following diagram:

$$
\begin{array}{cccc}
\text { continuity } \Rightarrow \begin{array}{c}
W K \text {-continuity } \\
\Downarrow
\end{array} & \Rightarrow & W O \text {-continuity } \\
\Downarrow & & \Downarrow \\
& g w_{\tau}^{*} \text {-continuity } & \Rightarrow & g w_{\tau} \text {-continuity } \\
\Uparrow & & \Uparrow \\
& g w_{\tau}^{*} \text {-irresolute } & \Rightarrow & g w_{\tau} \text {-irresolute }
\end{array}
$$

Theorem 4.9. Let $f:\left(X, w_{\tau}\right) \rightarrow\left(Y, w_{\mu}\right)$ be a function on two associated $w$-spaces with $\tau$ and $\mu$. Then the following statements are equivalent:

(1) $f$ is $g w_{\tau}$-irresolute.

(2) $f\left(g w_{\tau} C(A)\right) \subseteq g w_{\mu} C(f(A))$ for $A \subseteq X$.

(3) $g w_{\tau} C\left(f^{-1}(V)\right) \subseteq f^{-1}\left(g w_{\mu} C(V)\right)$ for $V \subseteq Y$.

(4) $f^{-1}\left(g w_{\mu} I(V)\right) \subseteq g w_{\tau} I\left(f^{-1}(V)\right)$ for $V \subseteq Y$

Proof. It is similar to the proof of Theorem 3.7.

Corollary 4.10. Let $f:\left(X, w_{X}\right) \rightarrow\left(Y, w_{Y}\right)$ be a function between $w$ spaces. Then the following statements are equivalent:

(1) $f$ is $g w_{\tau}$-irresolute.

(2) $f^{-1}(V)=g w_{\tau} I\left(f^{-1}(V)\right)$ for every $g w_{\mu}$-open set $V \in Y$.

(3) $f^{-1}(B)=g w_{\tau} C\left(f^{-1}(B)\right)$ for every $g w_{\mu}$-closed set $B \subseteq Y$.

Proof. From Theorem 2.2 and Theorem 4.9, it is obvious.

\section{References}

[1] Á. Csázár; Generalized Topology, Generalized Continuity, Acta Math. Hungar., 96 (2002), 351-357.

[2] D. C. Kent and W. K. Min; Neighborhood Spaces, International Journal of Mathematics and Mathematical Sciences, 32(7) (2002), 387-399.

[3] N. Levine; Generalized closed sets in topology, Rend. Cir. Mat. Palermo, 19(1970), 89-96. 
[4] N. Levine; Semi-open sets and semi-continuity in topological spaces, Ams. Math. Monthly, 70(1963), 36-41.

[5] H. Maki; On generalizing semi-open and preopen sets, Report for Meeting on Topological Spaces Theory and its Applications, August 1996, Yatsushiro College of Technology, 1318.

[6] A. S. Mashhour, M. E. Abd El-Monsef and S. N. El-Deeb; On precontinuous and weak precontinuous mappings, Proc. Math. and Phys. Soc. Egypt, 53 (1982), 47-53.

[7] W. K. Min; Some Results on Generalized Topological Spaces and Generalized Systems, Acta Math. Hungar., 108 (1-2) (2005), 171-181.

[8] W. K. Min; On Weak Neighborhood Systems and Spaces, Acta Math. Hungar., 121(3) (2008), 283-292.

[9] W. K. Min and Y. K. Kim; On Weak Structures and $w$-spaces, Far East Journal of Mathematical Sciences, 97(5) (2015), 549-561.

[10] W. K. Min and Y. K. Kim; WO-continuity and $W K$-continuity On Associated $w$-spaces; International Journal of Pure and Applied Mathematics, 102(2) (2015), 349 - 356.

[11] W. K. Min and Y. K. Kim; On generalized $w$-closed sets in associated $w$-spaces; submitted.

[12] O. Njastad; On some classes of nearly open sets, Pacific Journal of Mathematics, 15(3)(1964), 961-970.

[13] F. Siwiec; On Defining a Space by a Weak Base, Pacific Journal of Mathematics, 52(1) (1974), 351-357. 
